\section{(2) OPEN ACCESS}

\title{
Education level and outcomes after acute myocardial infarction in China
}

\author{
Xiqian Huo, ${ }^{1}$ Rohan Khera, ${ }^{2}$ Lihua Zhang, ${ }_{1}$ Jeph Herrin, ${ }^{3}$ Xueke Bai, ${ }^{1}$ Qianying Wang, \\ Yuan Lu, ${ }^{4}$ Khurram Nasir, ${ }^{4}$ Shuang $\mathrm{Hu}^{1}{ }^{1}$ Jing Li, ${ }^{1} \mathrm{Xi} \mathrm{Li},{ }^{1}$ Xin Zheng, \\ Frederick A Masoudi, ${ }^{5}$ John A Spertus, ${ }^{6}$ Harlan M Krumholz, ${ }^{3,4,7}$ Lixin Jiang $^{1}$
}

For numbered affiliations see end of article.

\section{Correspondence to}

Dr Lixin Jiang, National Clinical Research Center of Cardiovascular Diseases, Fuwai Hospital, Beijing 100037, China; jiangl@fwoxford.org

Xiqian Huo and Rohan Khera are joint first authors. Harlan M. Krumholz and Lixin Jiang are joint senior authors. Harlan M. Krumholz and Lixin Jiang are joint senior authors.

Received 18 June 2018 Revised 25 November 2018 Accepted 30 November 2018 Published Online First 19 January 2019

\section{ABSTRACT \\ Objective To assess the association between educational attainment and acute myocardial infarction (AMI) outcomes in China to inform future healthcare interventions. \\ Methods We used data from the China Patient-centred Evaluative Assessment of Cardiac Events-Prospective AMI study of 3369 consecutive patients hospitalised with AMI from 53 hospitals. Educational attainment was categorised as: high (senior high school, college or postgraduate degree), intermediate (junior high school) or low (primary school or illiterate). We used survival models to assess the relationship between education and 1-year major adverse cardiovascular events (MACE), all- cause mortality, both unadjusted and after adjustment for demographic characteristics and cardiovascular risk factors.}

Results The median participant age was 61 (52, 69 ) years, $23.2 \%$ were women, and $33.3 \%$ had high, $32.4 \%$ intermediate and $34.3 \%$ low educational attainment. In unadjusted analysis, compared with high educational attainment, low educational attainment was associated with a higher 1-year risk of MACE (HR 2.41, 95\% Cl 1.72 to 3.37) and death (HR for low vs high education $3.09,95 \% \mathrm{Cl} 1.69$ to 5.65). In risk-adjusted analyses, the association between education and death was attenuated and no longer statistically significant (adjusted HR 1.41, $95 \% \mathrm{Cl} 0.74$ to $2.69, \mathrm{p}=0.30)$. However, the risk of 1-year MACE (adjusted HR 1.68, 95\% Cl 1.18 to $2.41, p=0.004$ ) remained significantly greaterin low educational attainment group.

Conclusions In a national Chinese cohort of patients hospitalised with AMI, low educational attainment was associated with a higher risk of adverse events in the year following discharge. This association highlights the need to consider interventions to improve AMI outcomes in adults with low levels of education.

Clinical trial registration NCT01624909; Results.

\section{INTRODUCTION}

Delivering high-quality care equitably is an important aspiration of a high-performing health system. Disparities related to socioeconomic status are of particular concern. Several studies have identified sociodemographic disparities in the incidence of disease, prevalence of risk factors, life expectancy ${ }^{1-4}$ and disparities after acute healthcare events, a period of increased vulnerability. Among other sociodemographic factors, lower educational attainment is associated with limited access to healthcare, worse socioeconomic status and unhealthy lifestyle behaviours, ${ }^{56}$ all of which contribute excess risk for poor outcomes.

Given the important role of secondary prevention therapies and behavioural modification in improving outcomes, acute myocardial infarction (AMI) is an important condition to assess for disparities in post-acute care. It is plausible that education and other sociodemographic factors that are not directly related to the severity of an AMI may have an impact on outcomes. Understanding these relationships is particularly important in China, which is experiencing a growing burden of AMI and where levels of educational attainment vary widely. ${ }^{7}$ Moreover, China is in the midst of a healthcare reform that seeks to provide the population with greater access to high-quality care and essential medications. $^{89}$ If, despite the reform efforts, persistent disparities by education exist, then China may need to adopt different strategies to achieve greater equity. If present, it would be important to determine if disparities associated with education can be explained by other factors, such as cardiovascular risk profiles, that could potentially identify targets for future interventions to reduce such disparities. The evidence from Western nations is equivocal and is unlikely to generalise to the unique sociocultural and political landscape of China. ${ }^{10-13}$

Accordingly, using data from a large, national, prospective study, we sought to examine the association between educational attainment and clinical outcomes among Chinese patients hospitalised for $\mathrm{AMI}$ and to explore potential clinical factors that could potentially drive this association.

\section{METHODS}

\section{Data source and study population}

The design of the China Patient-centred Evaluative Assessment of Cardiac Events-Prospective AMI Study has been published previously. ${ }^{14}$ In brief, it is a large nationwide prospective study that consecutively recruited patients from 53 hospitals (35 tertiary and 18 secondary hospitals) throughout the 21 provinces in China between 2012 and 2015. Patients were eligible if they were $\geq 18$ years of age, hospitalised for AMI and presented within 24 hours of onset of symptoms. The diagnosis of AMI was consistent with the third universal definition. ${ }^{15}$ Patients were excluded if

To cite: Huo $\mathrm{X}$, Khera $\mathrm{R}$ 


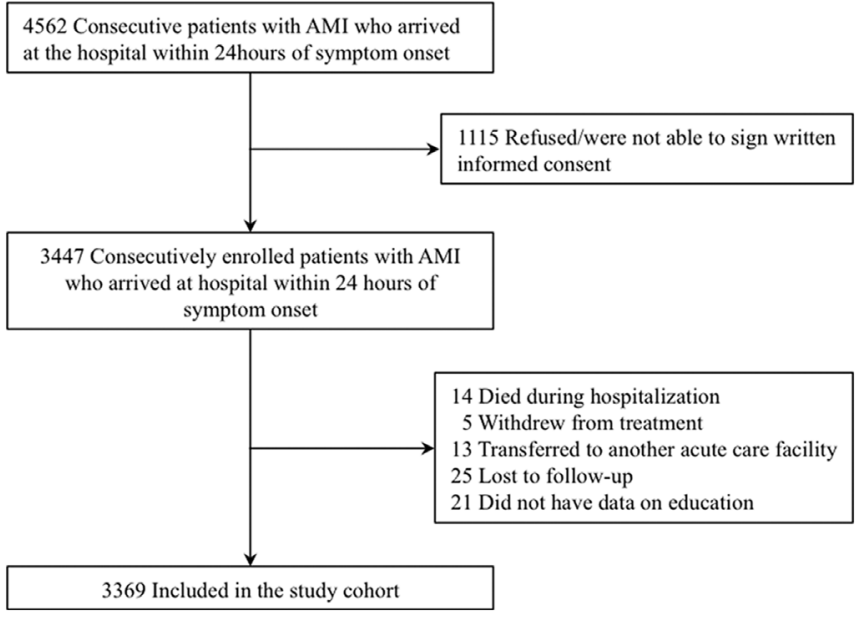

Figure 1 Flowchart of the study cohort. AMI, acute myocardial infarction.

they died or were withdrawn from treatment during hospitalisation $(n=19)$, were transferred to another facility $(n=13)$, were lost to follow-up at 1 year after discharge $(n=25)$ or did not have data on education $(\mathrm{n}=21)$. In total, 3369 eligible patients were included in our analysis, representing 97.7\% of the adults discharged alive following an AMI (figure 1). Enrolled patients completed a baseline interview during hospitalisation and were followed up at 1, 6 and 12 months postdischarge. All participants provided written informed consents at the initial visit.

The Chinese government including Ministry of Science and Technology and National Health and Family Planning Commission had no role in the study design, data collection, data analysis and interpretation, or in the preparation of the report. The study was registered at www.clinicaltrials.gov (NCT01624909).

\section{Education status}

We collected information on socioeconomic characteristics with a standardised questionnaire in an in-person interview. Education was self-reported as a five-level categorical variable ranging from illiterate to college/postgraduate. For reporting and analysis, we aggregated education categories into three levels: high (senior high school, college or postgraduate degree), intermediate (junior high school) or low (primary school or below) based on the distribution of education variable. The educational profile in our study was similar to the results of the latest national population sampling survey in China. ${ }^{16}$

\section{Patient characteristics}

We obtained demographic and clinical characteristics through abstraction of medical charts for the index hospitalisation, supplemented with patient interviews. Age and sex were collected as demographic information. Clinically relevant variables reflecting cardiovascular risk profile were selected based on a review of the literature. These included diabetes, hypertension, hyperlipidaemia, smoking status, family history of coronary heart disease (CHD), systolic blood pressure (SBP) and heart rate at admission, as well as evidence of prior cardiovascular diseases such as AMI, heart failure, angina and stroke. We included time to hospital arrival defined as the time between symptom onset and hospital arrival in hours using the documented timestamp of medical charts. As a marker of clinical severity on presentation, mini-GRACE risk score and left ventricular ejection fraction (LVEF) on initial presentation were also included. The mini-GRACE risk score is a modified and valid version of the GRACE risk score that includes variables for age, SBP at admission, ST-segment deviation on ECG, cardiac arrest at admission, elevated cardiac enzyme levels and heart rate at admission. ${ }^{17}$ We abstracted data on hospital treatments from medical charts, such as coronary angiography, revascularisation including percutaneous coronary intervention (PCI) or coronary artery bypass graft (CABG), thrombolytic therapy, inhospital and discharge medications.

\section{Outcomes}

The outcomes of interest in our study were major adverse cardiovascular event (MACE) and death from any cause within 1 year of discharge. MACE was defined as death from any cause, hospitalisations for non-fatal myocardial infarction, non-fatal stroke or heart failure. We ascertained major adverse events with the same approach used in our large international multicentre trials. ${ }^{18}$ We collected information on clinical events through follow-up interviews, and local clinic staff sought information systematically from relevant hospital records and death certificates. If a patient was unable or unwilling to attend the scheduled interview, information was collected over the telephone through a direct conversation between study physicians and patients or their relatives. All data were centrally adjudicated at the national coordinating centre by trained clinicians.

\section{Statistical analysis}

We examined differences in demographic and cardiovascular disease characteristics across education categories using Kruskal-Wallis test for trend of continuous variables (ie, age), and Mantel-Haenszel tests for trends of categorical variables (all other variables).

We compared 1-year MACE and mortality across education levels using Kaplan-Meier analysis and log-rank tests. In multivariable models, we evaluated the independent effect of education on 1-year MACE and mortality using survival analysis with frailty proportional hazards models, which explicitly accounted for clustering of patients within hospitals. ${ }^{19}$ We then evaluated for the effects of education in sequential multivariable models by adding sequential sets of covariates. Specifically, we first used Cox model to assess the relationship of outcomes with education (model 1) and then examined for changes in the association between education and outcomes after adjusting for demographic characteristics including age and sex (model 2). We then added covariates for cardiovascular clinical characteristics in addition to model 2 (model 3), in order to assess for the confounding effect of cardiovascular risk factors on the association between education and outcomes. Candidate variables of clinical characteristics in the model included diabetes, hypertension, hyperlipidaemia, smoking status, SBP at admission, heart rate at admission, AMI, heart failure, angina, stroke, family history of CHD, LVEF, mini-GRACE risk score, time from symptom to hospital arrival, inhospital coronary angiography, inhospital revascularisation and inhospital thrombolytic therapy. The proportional hazards assumption was checked for the full adjustment model. In sensitivity analyses, we assessed for the association between education and outcomes after combining low-education and intermediate-education groups or assessing education as a five-group variable.

In this study, follow-up for mortality and MACE was available for $99.3 \%$ of the patients. There were no missing data for the selected variables of age, sex and cardiovascular risk profiles. There 
Table 1 Demographic characteristics and cardiovascular risk profile among patients hospitalised with different education levels

\begin{tabular}{|c|c|c|c|c|c|}
\hline & $\begin{array}{l}\text { Total } \\
(\mathrm{n}=3369)\end{array}$ & $\begin{array}{l}\text { Low education } \\
(n=1154)\end{array}$ & $\begin{array}{l}\text { Intermediate } \\
\text { education }(n=1092)\end{array}$ & $\begin{array}{l}\text { High education } \\
(n=1123)\end{array}$ & P value \\
\hline \multicolumn{6}{|l|}{ Demographics characteristics } \\
\hline \multicolumn{6}{|l|}{ Age } \\
\hline Median age, year (IQR) & $61(52,69)$ & $66(60,74)$ & $59(50,66)$ & $57(49,67)$ & $<0.001$ \\
\hline Age $>65$ years $(\%)$ & $1298(38.5)$ & $674(58.4)$ & $309(28.3)$ & $315(28.0)$ & $<0.001$ \\
\hline Female (\%) & $781(23.2)$ & $494(42.8)$ & $185(16.9)$ & $102(9.1)$ & $<0.001$ \\
\hline Insurance & & & & & $<0.001$ \\
\hline Urban insurance $^{*}$ & $1960(58.2)$ & $446(38.6)$ & $593(54.3)$ & $921(82.0)$ & \\
\hline Rural cooperative medical service & $1204(35.7)$ & $649(56.2)$ & $424(38.8)$ & $131(11.7)$ & \\
\hline Other insurance & $205(6.1)$ & $59(5.1)$ & $75(6.9)$ & $71(6.3)$ & \\
\hline \multicolumn{6}{|l|}{ Cardiovascular risk profiles } \\
\hline \multicolumn{6}{|l|}{ Pre-existing heart disease (\%) } \\
\hline AMI & $267(7.9)$ & $81(7.0)$ & $88(8.1)$ & $98(8.7)$ & 0.131 \\
\hline CABG & $5(0.1)$ & $3(0.3)$ & $2(0.2)$ & $0(0.0)$ & 0.108 \\
\hline $\mathrm{PCl}$ & $233(6.9)$ & $58(5.0)$ & $62(5.7)$ & $113(10.1)$ & $<0.001$ \\
\hline Angina & $132(3.9)$ & $50(4.3)$ & $45(4.1)$ & $37(3.3)$ & 0.203 \\
\hline Heart failure & $230(6.8)$ & $100(8.7)$ & $61(5.6)$ & $69(6.1)$ & 0.016 \\
\hline Stroke & $556(16.5)$ & $224(19.4)$ & $180(16.5)$ & $152(13.5)$ & $<0.001$ \\
\hline \multicolumn{6}{|l|}{ Coronary risk factors } \\
\hline Diabetes mellitus (\%) & $782(23.2)$ & $249(21.6)$ & $256(23.4)$ & $277(24.7)$ & 0.081 \\
\hline Hypertension (\%) & $1876(55.7)$ & $657(56.9)$ & $580(53.1)$ & $639(56.9)$ & 0.975 \\
\hline Dyslipidaemia (\%) & $2584(76.7)$ & $869(75.3)$ & $834(76.4)$ & $881(78.5)$ & 0.076 \\
\hline Current smoker (\%) & $1969(58.4)$ & $545(47.2)$ & $706(64.7)$ & $718(63.9)$ & $<0.001$ \\
\hline Family history of CHD & $383(11.4)$ & $79(6.8)$ & $140(12.8)$ & $164(14.6)$ & $<0.001$ \\
\hline Median heart rate at admission (IQR) & $74(65,84)$ & $73.5(64,84)$ & $75(65,84)$ & $74(66,83)$ & 0.789 \\
\hline Median SBP at admission (IQR) & $130(114,146)$ & $130(114,150)$ & $130(114,146)$ & $128(114,144)$ & 0.222 \\
\hline Median mini-GRACE (IQR) & $129(110,146)$ & $139(120,154)$ & $126(109,143)$ & $125(106,141)$ & $<0.001$ \\
\hline LVEF on initial presentation (\%) & & & & & 0.005 \\
\hline$<0.4$ & $244(7.2)$ & $90(7.8)$ & $78(7.1)$ & $76(6.8)$ & \\
\hline$\geq 0.4$ & $2648(78.6)$ & $862(74.7)$ & $863(79.0)$ & $923(82.2)$ & \\
\hline LVEF unmeasured & 477 (14.2) & $202(17.5)$ & $151(13.8)$ & $124(11.0)$ & \\
\hline Time from symptom to hospital arrival $\leq 6$ hours & $2404(71.4)$ & $795(68.9)$ & $768(70.3)$ & 841 (74.9) & 0.002 \\
\hline \multicolumn{6}{|l|}{ Inhospital process measures, n (\%) } \\
\hline Coronary angiography & $2579(76.6)$ & $766(66.4)$ & 859 (78.7) & $954(85.0)$ & $<0.001$ \\
\hline Revascularisation ( $\mathrm{PCl}$ or $\mathrm{CABG})$ & $2350(69.8)$ & $702(60.8)$ & $774(70.9)$ & $874(77.8)$ & $<0.001$ \\
\hline Thrombolytic therapy & $332(9.9)$ & $133(11.5)$ & $110(10.1)$ & $89(7.9)$ & 0.004 \\
\hline Dual antiplatelet & $2644(78.5)$ & $880(76.3)$ & 869 (79.6) & $895(79.7)$ & 0.620 \\
\hline Beta-blocker & $2742(81.4)$ & $898(77.8)$ & $902(82.6)$ & $942(83.9)$ & $<0.001$ \\
\hline ACEI/ARB & $2472(73.4)$ & $832(72.1)$ & $809(74.1)$ & $831(74.0)$ & 0.303 \\
\hline Statins & $3319(98.5)$ & $1132(98.1)$ & $1078(98.7)$ & $1109(98.8)$ & 0.191 \\
\hline \multicolumn{6}{|l|}{ Medications at discharge, $\mathrm{n}(\%)$} \\
\hline Dual antiplatelet & $2301(68.3)$ & $773(67.0)$ & 737 (67.5) & 791 (70.4) & 0.078 \\
\hline Beta-blocker & $1727(51.3)$ & $545(47.2)$ & $554(50.7)$ & $628(55.9)$ & $<0.001$ \\
\hline ACEI/ARB & $1673(49.7)$ & $565(49.0)$ & $538(49.3)$ & $570(50.8)$ & 0.393 \\
\hline Statins & $2583(76.7)$ & $867(75.1)$ & 839 (76.8) & 877 (78.1) & 0.094 \\
\hline \multicolumn{6}{|l|}{ 1-year outcomes } \\
\hline 1-year death & $84(2.5)$ & $44(3.8)$ & $26(2.4)$ & $14(1.2)$ & $<0.001$ \\
\hline 1-year MACE & $250(7.4)$ & $126(10.9)$ & $75(6.9)$ & $49(4.4)$ & $<0.001$ \\
\hline
\end{tabular}

*Indicates any type of insurance for urban residents in China.

$A C E I$, angiotensin-converting enzyme inhibitor; $A M I$, acute myocardial infarction; $A R B$, angiotensin-receptor blocker; $C A B G$, coronary artery bypass grafting; $C H D$, coronary heart disease; LVEF, left ventricular ejection fraction; MACE, major adverse cardiovascular event; PCl, percutaneous coronary intervention; SBP, systolic blood pressure.

was no evidence of a significant interaction between age, sex and education.

All tests of significance were two-tailed, with a level of significance set at an alpha of 0.05 . Statistical analyses were performed using SAS (V.9.4).

\section{RESULTS}

\section{Baseline characteristics}

Our study sample included 3369 patients. The median age of the population was 61 years (range, 52-69), and 781 (23.2\%) were women. 1123 (33.3\%) had high, 1092 (32.4\%) intermediate 

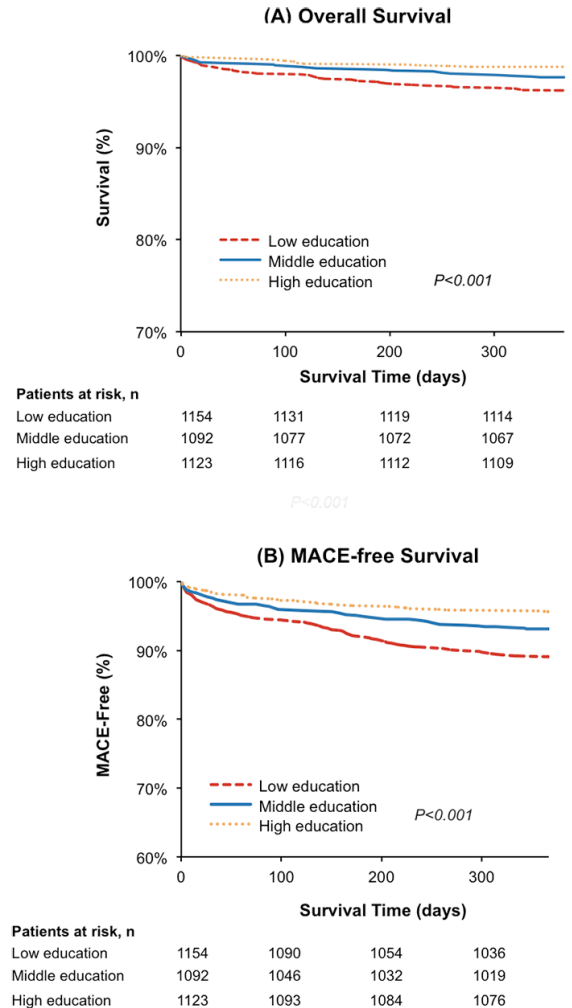

Figure 2 Unadjusted Kaplan-Meier curve. (A) Unadjusted KaplanMeier curve: 1-year all-cause mortality by educational attainment. (B) Unadjusted Kaplan-Meier curve: 1-year MACE by educational attainment. MACE, major adverse cardiovascular events.

and 1154 (34.3\%) had low educational attainment. Compared with patients with high educational attainment, those with a low educational attainment were older, more frequently women and more likely to have rural cooperative medical service $(p<0.001)$. Patients in the low educational attainment group had higher rates of heart failure and stroke, were less likely to smoke, had a lower LVEF at presentation and less frequently presented to the hospital within 6 hours after symptom onset than the middle and high educational attainment groups. In terms of the inhospital process measures, patients with a low educational attainment were less likely to receive coronary angiography and revascularisation and had higher rates of thrombolytic therapy. There were no significant differences of inhospital or discharge medication (eg, dual antiplatelet, statins, angiotensin-converting enzyme inhibitor/angiotensin receptor blocker) between diverse education groups (table 1 ).
Table 3 Categorising education into two groups and sequential risk adjustments on relationship

\begin{tabular}{|c|c|c|}
\hline \multirow[b]{2}{*}{ Risk adjustment category } & \multicolumn{2}{|c|}{ Low vs high education } \\
\hline & 1 year RR $(95 \% \mathrm{Cl})$ & $P$ value \\
\hline \multicolumn{3}{|l|}{ 1-year MACE } \\
\hline No adjustment (model 1) & 1.99 (1.45 to 2.73$)$ & $<0.001$ \\
\hline Demographic factors (model 2) & 1.61 (1.17 to 2.23$)$ & 0.004 \\
\hline $\begin{array}{l}\text { Demographic factors+CVD risk profiles } \\
\text { (model 3) }\end{array}$ & 1.61 (1.16 to 2.23$)$ & 0.004 \\
\hline \multicolumn{3}{|l|}{ 1-year mortality } \\
\hline No adjustment (model 1) & 2.52 (1.42 to 4.47$)$ & 0.002 \\
\hline Demographic factors (model 2) & $1.74(0.96$ to 3.14$)$ & 0.067 \\
\hline $\begin{array}{l}\text { Demographic factors+CVD risk profiles } \\
\text { (model 3) }\end{array}$ & $1.54(0.85$ to 2.81$)$ & 0.157 \\
\hline
\end{tabular}

Model 1, unadjusted;

model 2, adjusted for demographic characteristics including age and sex;

model 3, model two plus adjustment for cardiovascular clinical characteristics.

\section{Education and 1-year outcomes after AMI}

Kaplan-Meier survival curves for 1-year MACE and all-cause mortality by education group in subjects with AMI are shown in figure 2. Patients with lower educational attainment had higher MACE rates in the year after hospitalisation for AMI. Within 1 year after discharge, about $10.9 \%$ in the lowest education group occurred MACE compared with $4.4 \%$ of the highest education group $(\mathrm{p}<0.001)$. The 1 -year mortality varied significantly with educational attainment. In particular, 1-year mortality was over threefold higher in patients with the lowest educational attainment compared with those with the highest educational attainment $(3.8 \%$ vs $1.2 \%, \mathrm{p}<0.001)$.

Table 2 shows the relationship between educational attainment and 1-year adverse outcomes after sequentially adjusting for each set of covariates, and covariates meet the proportional hazards assumption. In survival analysis, lower educational attainment was strongly associated with higher 1-year MACE (HR for low vs high education $=2.41,95 \%$ CI 1.72 to 3.37 ). Even after adjusting for demographic and cardiovascular risk profiles, lower educational attainment was independently associated with a higher risk of MACE at 1 year compared with high educational attainment (risk-adjusted HR, 1.68; 95\% CI 1.18 to 2.41).

For 1-year mortality, patients with low education attainment were more likely to die than those with high educational attainment $(\mathrm{HR}=3.09,95 \% \mathrm{CI} 1.69$ to 5.65$)$. The addition of demographic factors attenuated the association $(\mathrm{HR}=1.70 ; 95 \% \mathrm{CI}$ 0.90 to 3.21 ), with little additional attenuation after adding cardiovascular risk factors $(\mathrm{HR}=1.41 ; 95 \% \mathrm{CI} 0.74$ to 2.69$)$.

Table 2 Sequential risk adjustments on the association between education and long-term outcomes after AMI

\begin{tabular}{|c|c|c|c|c|}
\hline \multirow[b]{2}{*}{ Risk adjustment category } & \multicolumn{2}{|c|}{ Intermediate vs high education } & \multicolumn{2}{|c|}{ Low vs high education } \\
\hline & 1 year RR $(95 \% \mathrm{Cl})$ & $P$ value & 1 year RR $(95 \% \mathrm{Cl})$ & $P$ value \\
\hline \multicolumn{5}{|l|}{ 1-year MACE } \\
\hline No adjustment (model 1) & 1.57 (1.09 to 2.25$)$ & 0.015 & 2.41 (1.72 to 3.37$)$ & $<0.001$ \\
\hline Demographic factors (model 2) & 1.54 (1.07 to 2.22$)$ & 0.019 & 1.68 (1.18 to 2.39$)$ & 0.004 \\
\hline Demographic factors+CVD risk profiles (model 3) & 1.54 (1.07 to 2.21$)$ & 0.021 & $1.68(1.18$ to 2.41$)$ & 0.004 \\
\hline \multicolumn{5}{|l|}{ 1-year mortality } \\
\hline No adjustment (model 1) & $1.92(1.00$ to 3.67$)$ & 0.050 & 3.09 (1.69 to 5.65$)$ & $<0.001$ \\
\hline Demographic factors (model 2) & $1.78(0.93$ to 3.42$)$ & 0.083 & $1.70(0.90$ to 3.21$)$ & 0.101 \\
\hline Demographic factors+CVD risk profiles (model 3) & $1.71(0.89$ to 3.30$)$ & 0.109 & $1.41(0.74$ to 2.69$)$ & 0.300 \\
\hline
\end{tabular}

Model 1, unadjusted; model 2, adjusted for demographic characteristics including age and sex; model 3, model 2 plus adjustment for cardiovascular clinical characteristics. 


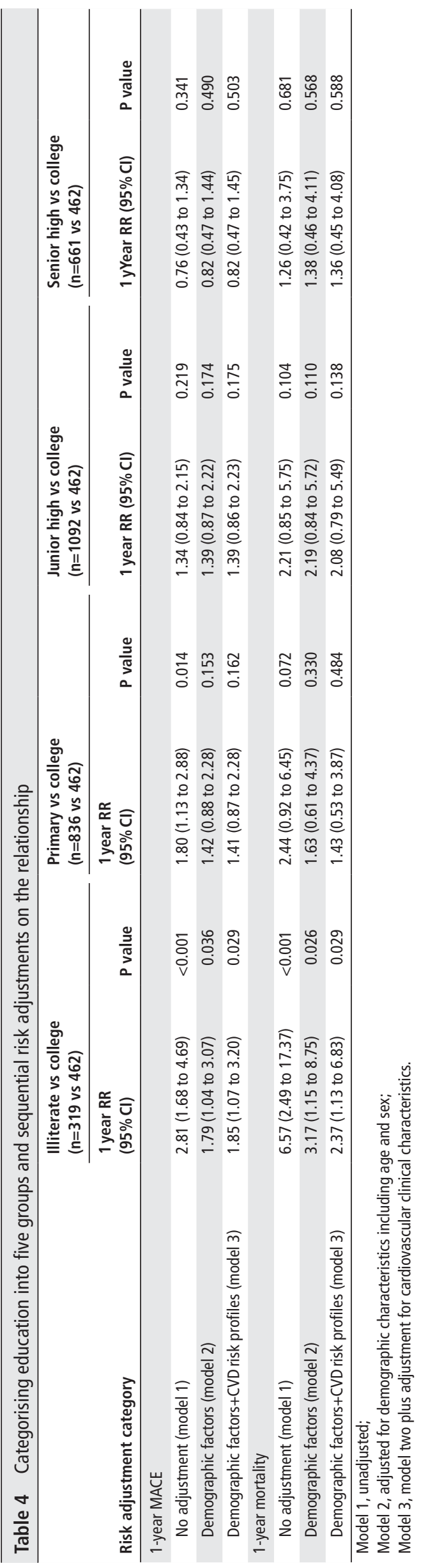

\section{Sensitivity analysis}

In sensitivity analysis, where educational attainment was categorised into two (high vs low and intermediate educational groups) and five classes (illiterate, primary school, junior high school, senior high school and college), education remained an independent predictor of MACE, consistent with the results of our primary analysis (tables 3 and 4).

\section{DISCUSSION}

In this first comprehensive report of the association between education and outcomes in a national cohort of patients with AMI in China, we found that patients with low educational attainment had a higher risk of adverse cardiovascular events and all-cause mortality in the 1-year period following their hospitalisation for AMI. Compared with senior high school, college or post-graduate degree, those with primary school education or less had a 2.4-fold higher rate of MACE. In the fully adjusted analysis, patients in the low education group had a 1.7 -fold higher risk of MACE in the year after AMI than those in high education group. No single risk factor was strongly associated with the outcomes.

Our study expands on previous literature on education and outcomes in several respects. First, we are not aware of any nationwide cohort studies of Chinese adults with AMI followed for a year. One previous Chinese study included an assessment of education for AMI outcomes and indicated that patients with middle or higher school education had lower inhospital mortality than those with primary school degree. However, the study mainly focused on inhospital mortality as the outcome, assessing educational attainment as one of the predictors for inhospital mortality among patients with AMI. ${ }^{20}$ Another study explored the association between socioeconomic status and financial burden among Chinese patients with CVD, but it did not provide evidence of patients' outcomes, thus we have no idea about the association between education and outcomes among patients with AMI in China. ${ }^{21}$ Second, we prospectively identified demographic and CVD risk factors that are relevant to the relationship between education and outcomes and found a clear association between low education and adverse cardiovascular outcomes. Studies from Europe and America have found an inconsistent association between education and adverse cardiovascular outcomes among patients with AMI based on their educational status. ${ }^{410-13} 22-24$ Some of this inconsistency arises from inconsistent assessment and inclusion of important cardiovascular risk factors such as hypertension, dyslipidaemia, smoking and pre-existing heart diseases in the assessment of these differences. ${ }^{11} 1322$

The mechanisms underlying the association between less education and higher risks for 1-year MACE remain unclear. We found that the higher rates of MACE among individuals with low educational attainment were attenuated after adjusting for demographic and comorbidity differences between these groups, with differences in demographic factors including age and sex accounting for a substantial proportion of differences in adverse outcomes between these two groups. Moreover, previous studies on education and health point to an array of social, environmental or genetic predisposition factors as being relevant to health outcomes. ${ }^{425}{ }^{26}$ Although adjustments in our analysis have been made, patients in different education groups may have more variations in baseline lifestyle choices and risk factors control due to different latency, and thus further study to fully capture the impact of these factors that extend beyond AMI would be imperative. Additionally, since these education-based 
differences in post-AMI outcomes still persisted after risk-adjustment, other factors, such as post-acute care and patient-related responses, may be playing an important role in driving these differences. In this respect, those with higher educational attainment may be more adept at post-discharge self-care and seek for the more optimal standard of care treatment. ${ }^{27}$ Highly educated patients may also have a better understanding of health knowledge and fewer financial barriers to accessing healthcare, leading to improved use of follow-up health services. Dedicated studies would need to focus on the role of follow-up care, medication adherence and the utilisation of rehabilitation services in understanding education-based differences in cardiovascular outcomes. Future targets for intervention in those with low educational attainment can then focus on improving the most challenging aspects of post-discharge care and more rigorous follow-up for these vulnerable patients. These findings could also stimulate policy and public health discussions to develop practical and sustainable strategies such as initiating closely and aggressive care coordination to provide more convenient health access to those populations and creating specific educational materials appropriate for their literacy. While there was a similar association with mortality, there were few deaths (84 deaths in low/high education groups) and our study was likely underpowered any differences between groups.

Several limitations should be considered in the interpretation of this study. First, this was an observational study, so we cannot assume that relationships are causal; there may be unobserved confounders that explain the relationship between education and outcomes. For example, we had limited information on door-to-balloon time and long-term medication usage, which all may be important in further understanding this association. Moreover, we did not explore other issues such as specifics on revascularisation (eg, non-culprit lesion information) after follow-up, while such information could help with hypotheses for the mechanism of the effect, if causal. Second, although the study was conducted in selected hospitals that represented their respective geographic regions, the results of the study may not be generalised to the whole population in China. However, the overall distribution of education-based groups, as well as outcomes among those with AMI, is consistent with those observed in national data from China. ${ }^{28}$ Third, education category in our study is different from some western studies that considered college or postgraduate as high educational attainment group. However, China has education patterns that are distinctive from western countries: in China, less than one thirds of population possess senior high school or above diploma, and only $13 \%$ of the citizens have achieved college or postgraduate degree according to latest national surveys, ${ }^{16}$ while $88 \%$ of the populations in USA completed at least high school education and more than one thirds have finished college degree at the same period..$^{29}$ The chance to get senior high school education in China is similar to getting college in USA or western countries, and with this respect, we choose the present categorisation as we did in the paper. Fourth, although we determined the definition of MACE based on the literature review, we included only the most important and relevant cardiovascular events as outcomes. Other events such as atrial fibrillation were not included in our analysis. Finally, education attainment was self-reported. However, it is unlikely that self-reported education is biased in ways that relate to health outcomes; for this reason, patient self-report has been used in all prior studies and in international studies and education status is considered reliable. ${ }^{112330}$

\section{CONCLUSION}

We found that less educated patients hospitalised for AMI have worse outcomes in China; efforts to reduce disparities of care in China must include a focus on identifying and mitigating barriers for this vulnerable patient group.

Keymessages

What is already known on this subject?

- In Western countries, there is an inconsistent association between educational attainment and clinical outcomes in patients following an acute myocardial infarction (AMI), however, data from China are limited.

\section{What might this study add?}

- In China, a country with a diverse population and wide variation in levels of education, we observed that patients in the 1-year period following their hospitalisation for AMI in comparison with patients with a senior high school, college or post-graduate degree, those with a primary school or lower education had a 2.4-fold higher rate of major adverse cardiovascular events, even after adjusting for potential confounders.

\section{How might this impact on clinical practice?}

- Given the substantial burden of cardiovascular disease and a global imperative to adopt strategies to achieve greater equity, these findings highlight the need to mitigate the excess risk experienced by those with limited education.

\section{Author affiliations}

${ }^{1}$ National Clinical Research Center of Cardiovascular Diseases, State Key Laboratory of Cardiovascular Disease, Fuwai Hospital, National Center for Cardiovascular

Diseases, Chinese Academy of Medical Sciences and Peking Union Medical College, Beijing, China

${ }^{2}$ Division of Cardiology, University of Texas Southwestern Medical Center at Dallas, Dallas, Texas, USA

${ }^{3}$ Section of Cardiovascular Medicine, Department of Internal Medicine, Yale University School of Medicine, New Haven, Connecticut, USA

${ }^{4}$ Center for Outcomes Research and Evaluation, Yale-New Heaven Hospital, New Heaven, Connecticut, USA

${ }^{5}$ Division of Cardiology, University of Colorado Anschutz Medical Campus, Aurora, Connecticut, USA

${ }^{6}$ Health Outcomes Research, Saint Luke's Mid America Heart Institude/University of Missouri-Kansas City, Kansas City, Missouri, USA

${ }^{7}$ Department of Health Policy and Management, Yale Univeristy School of Public Health, New Heaven, Connecticut, USA

Correction notice Since this paper was first published online first, figure 2 has been updated. The replacement version includes the survival times data under part $\mathrm{A}$ and $B$ of the figure.

Acknowledgements The authors appreciate the multiple contributions made by study teams at the China Oxford Centre for International Health Research and the Yale-New Haven Hospital Center for Outcomes Research and Evaluation in the realms of study design and operation, particularly the data collection and analysis by Hongzhao Zhang, Jiamin Liu, Wenchi Guan, Li Li, Xiaofang Yan, Ying Sun, Siying Niu, Qian Xiao, Xuekun Wu, Yuan Yu, Fang Feng, Ning Zhang, Qiulan Xie, Jia Li, Jianxin Zhang, Hao Dai, Hui Zhong, Liping Zhang, Yang Yang, Yan Gao, Siming Wang and Bin Wang. They are very grateful to Yun Wang from Harvard T.H Chan School of Public Health for his advice on the analyses and drafting of the manuscript. They thank the support provided by the Chinese government. They also appreciate the patients who participated in this study.

Contributors HMK and LJ: conceived the China PEACE prospective study and take responsibility for all aspects of it. JL, XL, XZ, FAM, JAS, HMK and LJ: participated in designing the study. XB, QW and SH: analysed the data. XH and RK: generated the first draft of manuscript, with further support from $L Z, J H, Y L, K N, J L, X L, X Z$, FAM, JAS, HMK and LJ. All authors provided critical revision of the paper, read and approved the final manuscript. 
Funding This project was supported by the National Key Research and Development Program (2017YFC1310801, 2017YFC1310803) from the Ministry of Science and Technology of China, the Central Public-Interest Scientific Institution Basal Research Fund (2017NL32002) from CAMS, the Research Special Fund for Public Welfare Industry of Health (201202025) from the National Health and Family Planning Commission of China, the CAMS Innovation Fund for Medical Science (2017-I2M-B\&R-02), the 111 project (B16005) from the Ministry of Education of China.

Competing interests HMK is the recipient of a research grant from Medtronic and Johnson \& Johnson, through Yale University, to develop methods of clinical trial data sharing; chairs a cardiac scientific advisory board for United Health; works under contract with the Centers for Medicare \& Medicaid Services to develop and maintain performance measures that are publicly reported; is a participant/ participant representative of the IBM Watson Health Life Sciences Board; is a member of the Advisory Board for Element Science and the Physician Advisory Board for Aetna; and is the founder of Hugo, a personal health information platform. FAM receives salary support from the American College of Cardiology for his role as the Chief Science Officer of the National Cardiovascular Data Registries. The authors declare no conflict of interest.

Patient consent Obtained.

Ethics approval The central ethics committee at the National Center for Cardiovascular Diseases, local ethics committees at participating hospitals, and the Yale University Institutional Review Board approved the China PEACE-Prospective AMl study.

Provenance and peer review Not commissioned; externally peer reviewed.

Open access This is an open access article distributed in accordance with the Creative Commons Attribution Non Commercial (CC BY-NC 4.0) license, which permits others to distribute, remix, adapt, build upon this work non-commercially, and license their derivative works on different terms, provided the original work is properly cited, appropriate credit is given, any changes made indicated, and the use is non-commercial. See: http://creativecommons.org/licenses/by-nc/4.0/.

\section{REFERENCES}

1 Kershaw KN, Droomers M, Robinson WR, et al. Quantifying the contributions of behavioral and biological risk factors to socioeconomic disparities in coronary heart disease incidence: the MORGEN study. Eur J Epidemio/ 2013;28:807-14.

2 Nordahl H, Rod NH, Frederiksen BL, et al. Education and risk of coronary heart disease: assessment of mediation by behavioral risk factors using the additive hazards model. Eur J Epidemiol 2013;28:149-57.

3 Niu S, Zhao D, Zhu J, et al. The association between socioeconomic status of high-risk patients with coronary heart disease and the treatment rates of evidence-based medicine for coronary heart disease secondary prevention in China: Results from the Bridging the Gap on CHD Secondary Prevention in China (BRIG) Project. Am Heart J 2009;157:709-15.

4 Davies NM, Dickson M, Davey Smith G, et al. The causal effects of education on health outcomes in the UK Biobank. Nat Hum Behav 2018;2:117-25.

5 Alter DA, Iron K, Austin PC, et al. Socioeconomic status, service patterns, and perceptions of care among survivors of acute myocardial infarction in Canada. JAMA 2004;291:1100-7

6 Wilson DK, Kirtland KA, Ainsworth BE, et al. Socioeconomic status and perceptions of access and safety for physical activity. Ann Behav Med 2004;28:20-8.

7 Ralston DA, Holt DH, Terpstra RH, et al. The impact of natural culture and economic ideology on managerial work values: a study of the United States, Russia, Japan, and China. J Int Bus Stud 1997:28:177-207.

8 Chen Z. Launch of the health-care reform plan in China. Lancet 2009:373:1322-4.
9 The Lancet. What to expect for China's health in the future. Lancet 2017;389:226.

10 Rasmussen JN, Rasmussen S, Gislason GH, et al. Mortality after acute myocardial infarction according to income and education. J Epidemiol Community Health 2006;60:351-6.

11 Mehta RH, O'Shea JC, Stebbins AL, et al. Association of mortality with years of education in patients with ST-segment elevation myocardial infarction treated with fibrinolysis. J Am Coll Cardiol 2011;57:138-46.

12 Kirchberger I, Meisinger C, Golüke H, et al. Long-term survival among older patients with myocardial infarction differs by educational level: results from the MONICA KORA myocardial infarction registry. Int J Equity Health 2014;13:19.

13 Igland J, Vollset SE, Nygård OK, et al. Educational inequalities in 28 day and 1-year mortality after hospitalisation for incident acute myocardial infarction-a nationwide cohort study. Int J Cardiol 2014;177:874-80.

14 Du X, Pi Y, Dreyer RP, et al. The china patient-centered evaluative assessment of cardiac events (PEACE) prospective study of percutaneous coronary intervention: Study design. Catheter Cardiovasc Interv 2016;88:E212-E221.

15 Thygesen $\mathrm{K}$, Alpert JS, Jaffe AS, et al. Third universal definition of myocardial infarction. Circulation 2012;126:2020-35.

16 China NBoSotPsRo. 1\% national population sampling survey report, 2015.

17 Simms AD, Reynolds S, Pieper K, et al. Evaluation of the NICE mini-GRACE risk scores for acute myocardial infarction using the Myocardial Ischaemia National Audit Project (MINAP) 2003-2009: National Institute for Cardiovascular Outcomes Research (NICOR). Heart 2013;99:35-40.

18 HPS2-THRIVE Collaborative Group. HPS2-THRIVE randomized placebo-controlled trial in 25673 high-risk patients of ER niacin/laropiprant: trial design, pre-specified muscle and liver outcomes, and reasons for stopping study treatment. Eur Heart $J$ 2013;34:1279-91.

19 Pankratz VS, de Andrade M, Therneau TM. Random-effects Cox proportional hazards model: general variance components methods for time-to-event data. Genet Epidemiol 2005;28:97-109.

20 Li M, Li S, Du X, et al. Factors attributed to the higher in-hospital mortality of ST elevation myocardial infarction patients admitted during off-hour in comparison with those during regular hour. PLoS One 2017;12:e0175485.

21 Li C, Young BR, Jian W. Association of socioeconomic status with financial burden of disease among elderly patients with cardiovascular disease: evidence from the china health and retirement longitudinal survey. BMJ Open 2018;8:e018703.

22 Consuegra-Sánchez L, Melgarejo-Moreno A, Galcerá-Tomás J, et al. Educational level and long-term mortality in patients with acute myocardial infarction. Rev Esp Cardiol 2015;68:935-42

23 Alter DA, Chong A, Austin PC, et al. Socioeconomic status and mortality after acute myocardial infarction. Ann Intern Med 2006;144:82-93.

24 Bernheim SM, Spertus JA, Reid KJ, et al. Socioeconomic disparities in outcomes after acute myocardial infarction. Am Heart J 2007:153:313-9.

25 Tillmann T, Vaucher J, Okbay A, et al. Education and coronary heart disease: mendelian randomisation study. BMJ 2017:358:j3542.

26 Adler NE, Newman K. Socioeconomic disparities in health: pathways and policies Health Aff 2002;21:60-76.

27 Dennison CR, McEntee ML, Samuel L, et al. Adequate health literacy is associated with higher heart failure knowledge and self-care confidence in hospitalized patients. J Cardiovasc Nurs 2011;26:359-67.

28 Guo J, Li W, Wang Y, et al. Influence of socioeconomic status on acute myocardial infarction in the Chinese population: the INTERHEART China study. Chin Med J 2012;125:4214-20.

29 Bureau USC. Educational Attainment in the United States: 2015 - Census, 2015.

30 Seeman T, Merkin SS, Crimmins E, et al. Education, income and ethnic differences in cumulative biological risk profiles in a national sample of US adults: NHANES III (1988-1994). Soc Sci Med 2008;66:72-87. 